\title{
Design Principles for Soft-Rigid Hybrid Manipulators
}

Utku Culha $^{1}$, Josie Hughes ${ }^{2}$, Andre Rosendo ${ }^{2}$, Fabio Giardina ${ }^{2}$ and Fumiya Iida ${ }^{2}$

${ }^{1}$ Department of Mechanical and Process Engineering, ETH Zurich, Switzerland

${ }^{2}$ Biologically Inspired Robotics Laboratory, Department of Engineering, University of Cambridge, United Kingdom

Abstract In nature, manipulators have evolved into different morphologies with varying rigidity to accomplish different tasks. Soft and continuum tentacles of the octopus, rigid and strong pincers of the crab and ligamentous jointed fingers of the human demonstrate the relationship between the complexity of a host's task space and the design of its manipulator. Thus, the purpose of use a robotic manipulator should be considered as an important design parameter which governs the choice of appropriate materials and design rules. For tasks which require delicacy and strength at the same time, such as human-machine interaction, agriculture or robotic surgery hybrid soft-rigid manipulator designs should be investigated. Here, we present four design principles for building hybrid robot manipulators which incorporate soft and rigid materials and demonstrate each principle with working examples.

\section{Introduction}

Interaction with the environment is essential for the survival of intelligent life in nature. Especially animals have developed a great variety of manipulators with different morphology and rigidity which yields the necessary functionality to achieve life sustaining, interaction based tasks. For example, an octopus thrives in its habitat by utilizing its highly deformable soft octopus arms [1], whereas in comparison, a lobster has rigid dexterous pincers with thick strong exoskeletons [2]. Human hands lie in the middle of this spectrum in terms of the rigidity of their structure. The rigid bone phalanges and the soft ligamentous joints contribute to the overall strength while also providing the necessary flexibility of the human fingers [3].

For decades robotics research has focused on the design of robotic manipulators with rigid structures for use in industrial applications which require high precision, large force exertion and low mechanical flexibility [4]. Commonly used two or three fingered manipulators have led to the development of more anthropomorphic robotic manipulator designs such as Utah / MIT Hand [5] and DLR / HIT Hand II [6] which utilised rigid body links and fixed degree of freedom (DOF) joints. Recently there has been a shift towards bio-inspired manipulator designs which behave like or consist of continuum body soft materials. Advancing from Hirose's rigid link manipulator which behaves like a soft arm [7], later examples started using soft materials and continuum bodies. Manipulators such as Ilievski's soft gripper [8], the Octopus Arm Project [9] and RBO Hand 2 [10] are successful examples to manipulators with soft materials which exercise large deformations and structural conformity due to their high DOF.

\section{Hybrid Manipulator Definition}

By definition, hybrid is the combination of two different elements and it is typically used to define manipulators which combine parallel and serial mechanisms in robotics [11]. However, we are using hybrid to describe manipulators which combine soft and rigid materials. Figure 1 is an overview of the manipulator design space according to the choice of materials. Soft continuum body manipulators exploit large deformation capacities; demonstrating features such as higher DOF and structural compliance. Conversely, manipulators with interconnected rigid body links utilise rigid body dynamics and inverse kinematics enabling precise motions and exertion of higher magnitudes of forces. 


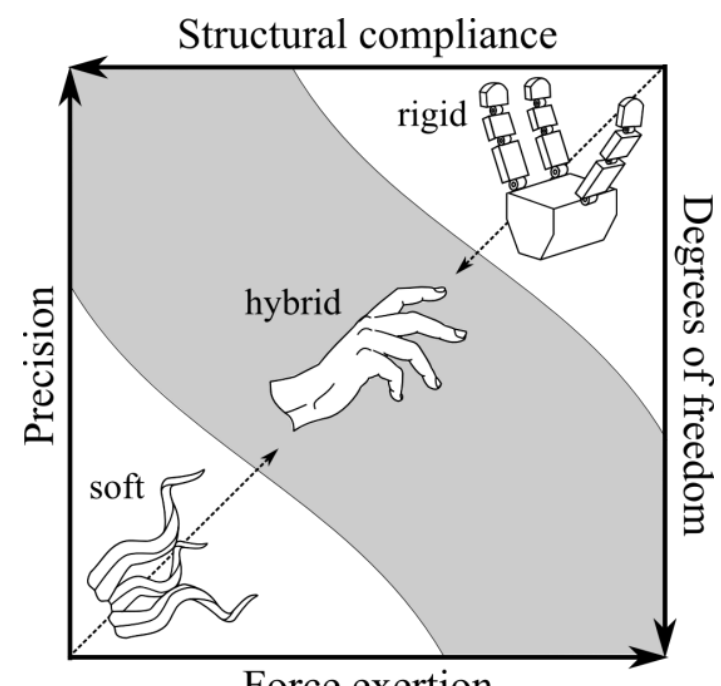

Force exertion

Figure 1. The position of soft, hybrid and rigid manipulators in the 2D design space. Quantitative change of the four important manipulator features: precision, structural compliance, degrees of freedom (DOF) and force exertion are shown on the axes. Hybrid manipulators lie on the fairly unexplored diagonal design space (grey coloured).

The hybrid manipulator lies in the middle of the design space as it is a combination of soft and rigid materials. Manipulators built this way can benefit from a sufficient proportion of all the features granted by both of the material types as shown in Figure 1. A key example found in nature would be the human hand which demonstrates impressive dexterity. Inspired from human fingers' ligamentous joints which connect rigid bones with soft tissues, robotic platforms such as Pisa/IIT Hand [12], iHY Hand [13] and the derivation of the ACT Hand [14] have been developed. Hybrid manipulators built similar to human hands can be promising candidates for achieving dexterous tasks in human-machine interaction (e.g. caretaking and rehabilitation), agriculture (e.g. planting and harvesting), invasive surgery and tactile exploration (e.g. sea/ocean bed and planetary surface investigations). However, human hand design is still very complicated and cannot be replicated with a top-down approach. That is why in the next section, we are going to present a bottom-up approach and list four design principles which can be utilised to build hybrid manipulators.

\section{Design Principles of Hybrid Manipulators}

In this section we present four design principles which are essential for the design of hybrid manipulators in order of increasing complexity with an example of a robotic manipulator given for each. Each manipulator design builds upon those presented previously, with the final manipulator encompassing all four principles.

\subsection{Articulated Link Structure}

Being able to generate form and force closure on objects enables manipulators to achieve complex interactions [15]. While the form closure guarantees the immobilisation of an object via geometric enclosure, the force closure allows in-hand manipulation. Articulated links with a hybrid usage of rigid and soft materials can allow a manipulator to be flexible and generate both types of closures simultaneously, as the soft articulation enables the rigid links to enclose the object and exert forces. Counter examples, such as the universal gripper [16] with an impressive grasping adaptivity through mainly form closure, cannot perform in hand manipulation due to its lack of articulated links. Similarly, the industrial manipulators without deformable link structures [4] can maintain a large scale of force closures, but cannot enclose objects which hinder their flexibility of grasping. 
(a)

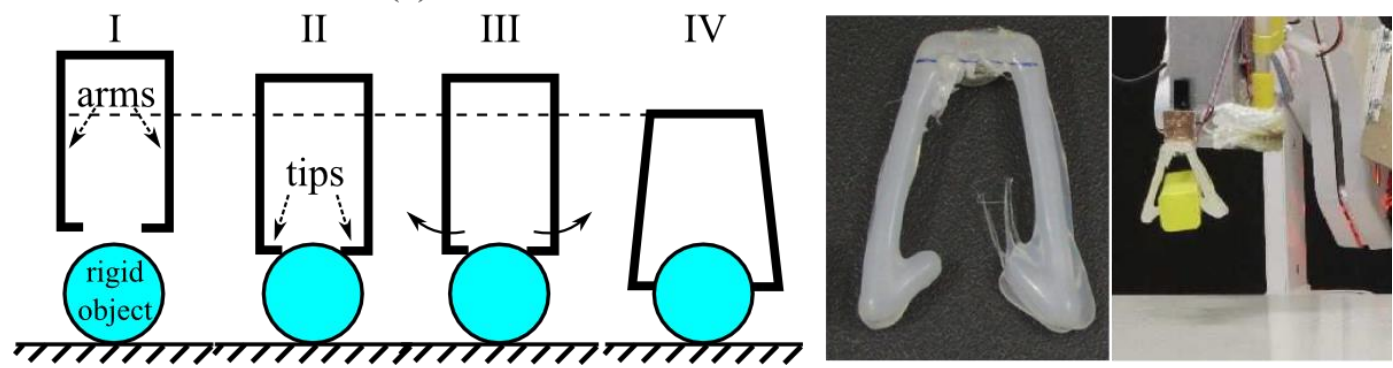

Figure 2. The simple design of the passive soft gripper allows the fingers to bend outwards when pushed against a rigid object to exploit the elasticity of the articulated link structure (a). Hot melt adhesive (HMA) is used to fabricate this simple design [17] and a robotic arm is used for pick and place tasks exploiting the environmental niche [18] (b).

The passive gripper shown in Figure 2(a) is a simple demonstration of articulated links. The arms are fabricated thinner and softer compared to the denser, more rigid tips, making the manipulator structurally hybrid as seen in Figure 2(b). This allows the gripper to elastically enclose the object while exerting forces at the tips.

\subsection{Soft Actuation - Tendon Driven Links}

It is necessary to have low inertia for a hybrid manipulator to achieve delicate and soft interactions. This requires the actuation mechanism to transfer forces to the target actuation point without hindering the softness of the manipulator. There are two types of candidate mechanisms which can meet this requirement. Pneumatichydraulic soft chambers bend continuum link structures by inflating, and merge links and joints together which may not be preferred in hybrid manipulators. In comparison, our preferred solution tendon drive systems allow transfer of force from a distant actuator over manipulator's rigid links, keeping the moment of inertia low. The tendon cables (e.g. kite lines) have a very high tensile strength on their longitudinal axis, but they are easily bendable on other directions, allowing complex routing over a hybrid manipulator structure. As tendons generate only pulling forces, counteracting forces can be achieved by using antagonistic tendon pairs or through the inherent elasticity of the joints.
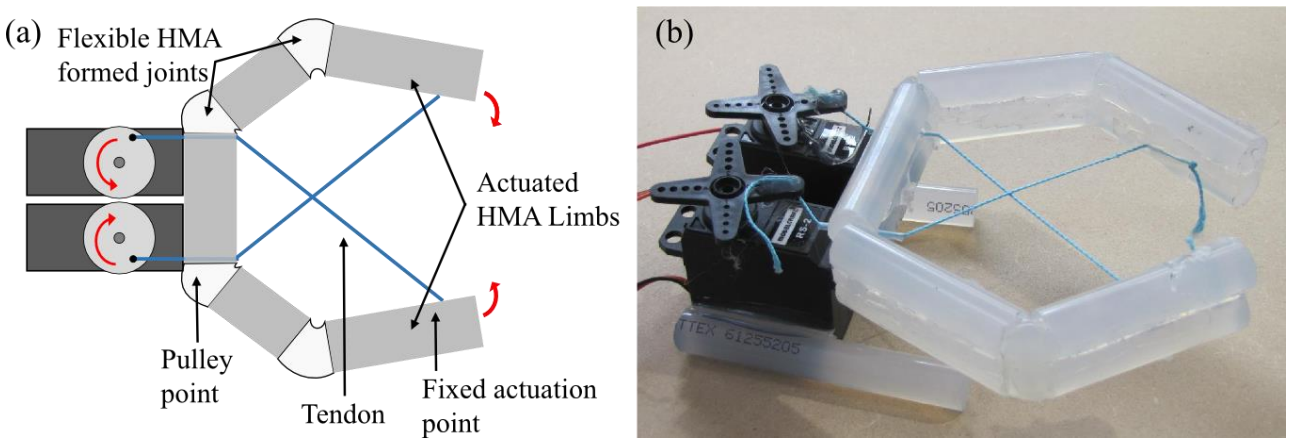

Figure 3. An improved version of the passive gripper design allows the usage of simple tendon actuation to perform finger flexion operation (a). Fingers are cold HMA blocks glued together with the melt HMA creating soft-joints which do not have a fixed axis of rotation such as hinges or gimbals (b).

Figure 3 shows the addition of tendons to a manipulator constructed from HMA material. Tendons allow the actuation source to be away from the gripper ends without distorting the soft body. Tendons are pulled to flex the links and relaxed to let the joint elasticity to bring the links back to their original resting position.

\subsection{Differential Stiffness via Multi-Material Usage}

The hybridity of soft and rigid sections can also be achieved through combining materials with differing mechanical stiffness. When exposed to an internal or external stimulus, a hybrid manipulator's lower stiffness 
parts will generate a larger motion compared to the higher stiffness parts. In other words, we can define the motion of a manipulator by using materials with differing stiffness. In biology, mechanical properties of every tissue are special for the functions they take part in. Similarly, in hybrid robotic manipulators, high tensile strength cables can be used as tendons, durable and flexible materials as joints, and strong materials as links.
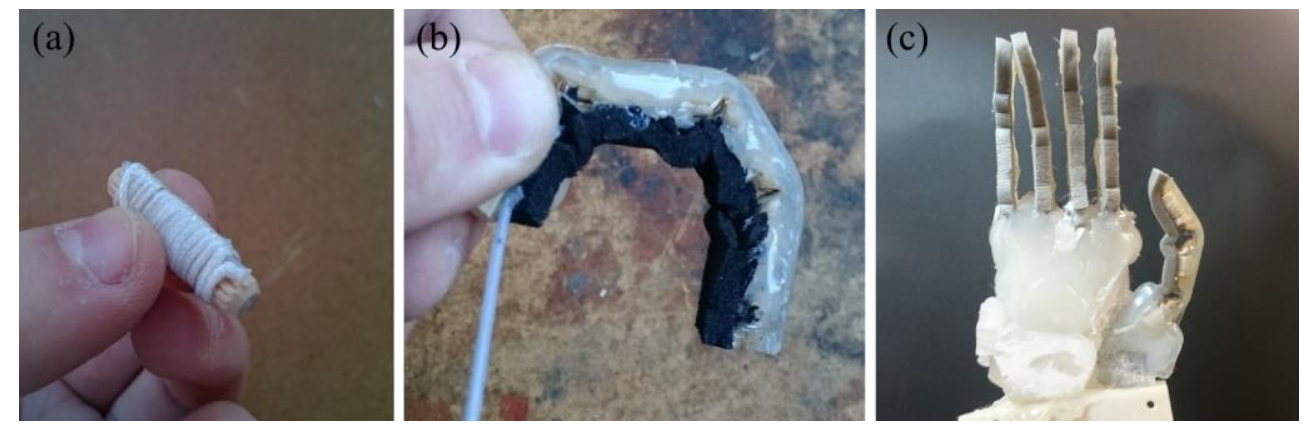

Figure 4. In this design, links are made of wood which carry the pulleys that allow the translation of actuator force through the fingers (a). Wood links are connected to each other with soft HMA material to form soft joints and contribute to the overall flexibility of the manipulator. Finger pads are covered with soft sponge materials to assist form closure (b). By repeating the same finger design, a hybrid manipulator that resembles the human hand is created. Palm is reinforced with rigid materials for the fingers to apply necessary forces during grasping (c).

Differential stiffness is demonstrated in the hybrid manipulator design in Figure 4. Tendons with high tensile strength are routed over the wooden links through the frictionless pulley tubes. The flexibility of the soft HMA joints contributes to the structural compliance and the high DOF motion of the fingers during interactions within the workspace. Additionally, the wooden links and the pulleys allow the transfer of actuator force carried by the tendons to the fingertips and generate successful grasping of objects.

\subsection{Guided Soft Joints}

Typical hinge and gimbal mechanisms are compliant only on the axes of their fixed degree of motion. Although these joints are easy to actuate and control, they provide little to the overall adaptability of the manipulators. Therefore a soft joint which can move in multiple directions would be suitable for improved structural compliance. However it is challenging to design such a joint by only using soft and deformable materials. Soft materials have infinite DOF which will make joints bend in undesired directions during force exertion and impossible to fully actuate with finite number of actuators. However, guiding soft joints with the geometry of the link end regions and the routing of the tendons can establish a controlled actuation. Links with guided soft joints can move and exert forces in predefined axes, but they can flex and adapt to the environment when disturbances are larger than a certain threshold. This makes the manipulators more adaptive and durable.
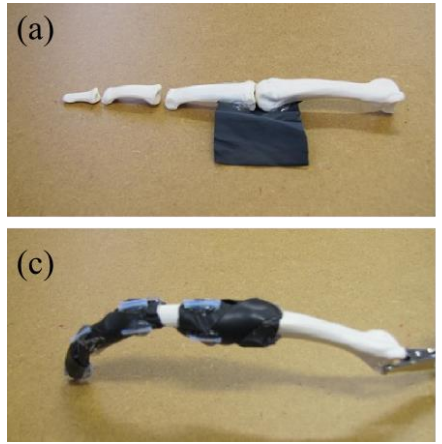

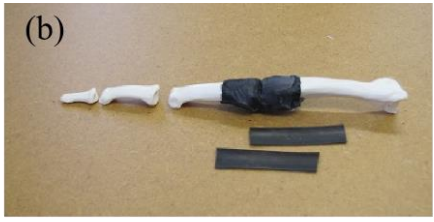

(d)

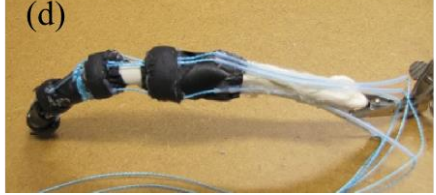

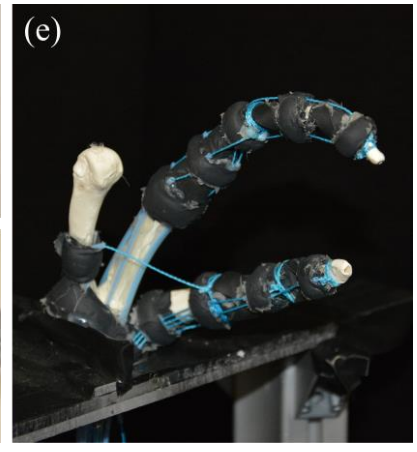

Figure 5. In this hybrid manipulator design [19] anatomically correct human finger bones are used as rigid parts and are connected to each other by two types of soft and elastic rubber elements to form the ligamentous structure of human finger joints (a-b). Tendon pulleys are attached on the rigid links and strong tendons are routed through the 
finger structure from these pulleys (c-d). The overall hybrid manipulator design uses 14 tendons to actuate the thumb and index finger for flexion, extension, abduction and adduction motions (e).

The manipulator shown in Figure 5 is the last example of a hybrid manipulator design. In addition to the embodiment of the previously shown principles, this anthropomorphic design uses the geometry of the bone cavity and the tendon routings to guide the soft joints [19]. The soft joints are made of two types of elastic rubber materials which resemble the ligamentous structure of the human finger joints.

\section{Discussion}

Hybrid manipulators are going to be central to the future development of safe, highly functional and adaptive systems which can work in environments which require safe and compliant interactions as shown in Figure 6. Clear applications for both soft and hard manipulators already exist, and the hybrid approach would not only solve problems that are not currently solved by these two, but would also overlap with these in some areas by offering additional precision/compliance due to the variable rigidity of the system. With the gradual automation of industrial and agricultural processes, a higher degree of compliance will enable a safer handling of delicate products, while current soft solutions can benefit from the advent of hybrid manipulators to improve their intrinsic speed/precision.
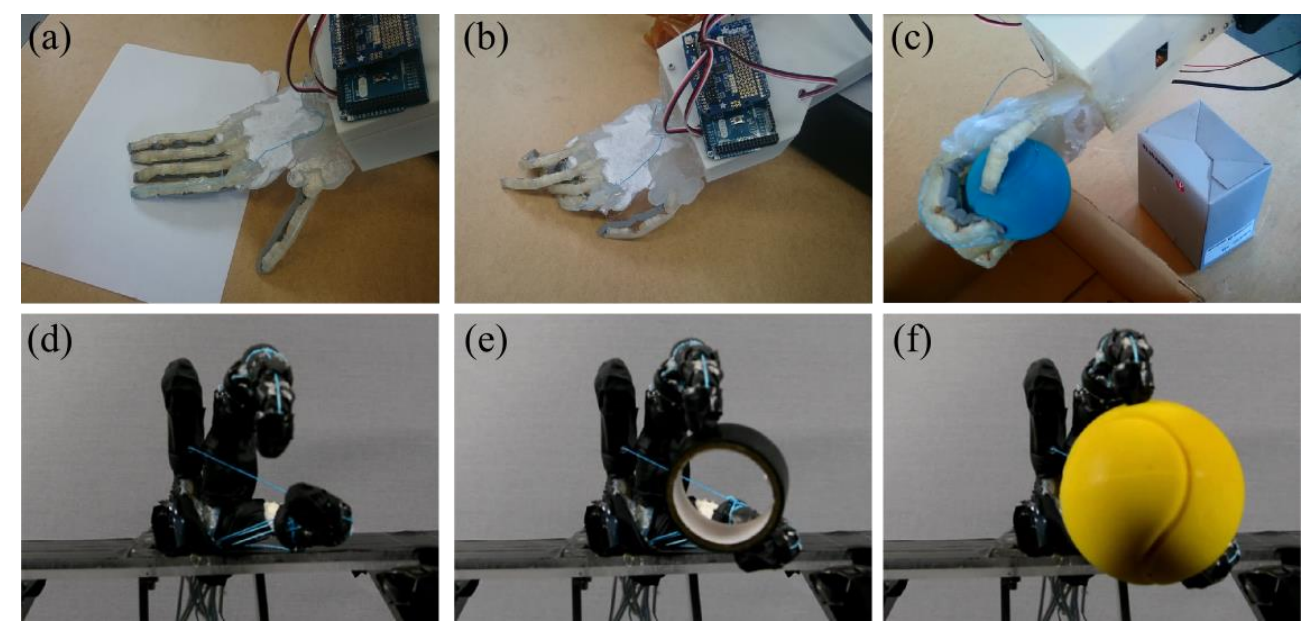

Figure 6. The structural compliance of the hybrid manipulators will allow them to use environmental niches to assist their tasks (a) and increase their durability in unexpected interactions (b). By using rigid structures and flexible joints, these manipulators can apply the necessary forces while adapting to the shapes of the objects they are handling (c-f).

Our better understanding of hybrid manipulators is dependent on a stronger academic push which addresses the challenges associated with soft and hard materials. Soft materials' inherent ability to deform in virtually infinite DOF can be useful in achieving highly deformable and adaptable manipulators. However, estimating the body state of these manipulators with a finite number of sensors has theoretical boundaries. Using guided soft joints [19] or custom sensor morphologies [20] may reduce the number of necessary sensors to detect soft deformations; however a deeper investigation is still very crucial. Additionally, assembly of multiple materials in a continuous way is a unique challenge for hybrid manipulators. With the increase of complexity and inclusion of more materials, the fabrication method switches from automation to assembly by hand as can be seen from the examples given. However, with the projected usage of hybrid manipulators in a large range of applications, automated manufacturing methods which combine multiple materials must be explored. These methods need to produce hybrid manipulators ensuring continuum connections between rigid and soft parts. This will require an interdisciplinary research on manufacturing and material science. 


\section{References}

[1] Kier WM, Stella MP (2007) The arrangement and function of octopus arm musculature and connective tissue. Journal of Morphology, 268(10): 831-843.

[2] Zhou F, Wu Z, Wang M, Chen K (2010) Structure and mechanical properties of pincers of lobster (Procambarus Clarkii) and crab (Eriocheir Sinensis). Journal of the Mechanical Behaviour of Biomedical Materials, 3(6):454-463.

[3] Kapandji IA (1987) The physiology of the joints: lower limb vol. 2. Churchill Livingstone, Edinburgh.

[4] Nof SY (1999) Handbook of industrial robotics vol. 1. John Wiley \& Sons, Toronto.

[5] Jacobsen SC, Iversen EK, Knutti DF, Johnson RT, Biggers KB (1986) Design of the Utah/MIT dextrous hand. Proc. IEEE Int. Conf. on Robotics and Automation (ICRA), 1520-1532.

[6] Liu H et al (2008) Multisensory five-finger dexterous hand The DLR/HIT Hand II. IEEE/RSJ Int. Conf. on Intelligent Robots and Systems (IROS), 3692-3697.

[7] Hirose S, Umetani Y (1978) The development of soft gripper for the versatile robot hand. Mechanism and Machine Theory, 13(3):351-359.

[8] Ilievski F, Mazzeo AD, Shepherd RF, Chen X, Whitesides GM (2011) Soft robotics for chemists. Angewandte Chemie, 123(8):1930-1935.

[9] Laschi C, Cianchetti M, Mazzolai B, Margheri L, Follador M, Dario P (2012) Soft robot arm inspired by the octopus. Advanced Robotics, 26(7):709-727.

[10] Deimel R, Brock O (2016) A novel type of compliant and underactuated robotic hand for dexterous grasping. International Journal of Robotics Research, 35(1-3):161-185.

[11] Tanev TK (2000) Kinematics of a hybrid (parallel-serial) robot manipulator. Mechanism and Machine Theory, 35(9):1183-1196.

[12] Catalano MG, Grioli G, Farnioli E, Serio A, Piazza C, Bicchi A (2014) Adaptive synergies for the design and control of the Pisa/IIT SoftHand. The International Journal of Robotics Research, 33(5):768-782.

[13] Odhner L U et al (2014) A compliant, underactuated hand for robust manipulation. The International Journal of Robotics Research, 33(5):736-752.

[14] Xu Z, Kumar V, Matsuoka Y, Todorov E (2012) Design of an anthropomorphic robotic finger system with biomimetic artificial joints. IEEE RAS\&EMBS Int. Conf. Biomedical Robotics and Biomechatronics (BioRob), $568-574$.

[15] Yoshikawa T (1999) Passive and active closures by constraining mechanisms. Journal of Dynamic Systems, Measurement, and Control, 121(3):418-424.

[16] Brown E, Rodenberg N, Amend J, Mozeika A, Steltz E, Zakin MR, Lipson H, Jaeger HM (2010) Universal robotic gripper based on the jamming of granular material. Proceedings of the National Academy of Sciences, 107(44):18809-18814.

[17] Wang L, Brodbeck L, Iida F (2014) Mechanics and energetics in tool manufacture and use: a synthetic approach. Journal of the Royal Society Interface, 11(100):20140827.

[18] Brodbeck L, Iida F (2012) Enhanced robotic body extension with modular units. IEEE/RSJ Int. Conf. on Intelligent Robots and Systems (IROS), 1428-1433.

[19] Culha U, Iida F (2016) Enhancement of finger motion range with compliant anthropomorphic joint design. Bioinspiration \& Biomimetics, 11(2):026001.

[20] Culha U, Nurzaman SG, Clemens F, Iida F (2014) SVAS3: strain vector aided sensorization of soft structures. Sensors, 14(7): 12748-12770. 